\title{
The Changing Epidemiology of Carbapenemase-Producing Enterobacterales
}

\author{
Khetam Hussein, M.D..,2*, Yuval Geffen, Ph.D., ${ }^{2,3}$, Orna Eluk, R.N. ${ }^{1}$, Sigal \\ Warman, R.N. ${ }^{1}$, Worood Aboalheja, R.N. ${ }^{1}$, Tamar Alon, R.N. ${ }^{1}$, Ibrahim Firan, \\ R.N. ${ }^{1}$, and Mical Paul, M.D. ${ }^{1,2}$ \\ ${ }^{\prime}$ Infectious Diseases Institute, Rambam Health Care Campus, Haifa, Israel; ${ }^{2}$ The Ruth E Bruce Rappaport \\ Faculty of Medicine, Technion-Israel Institute of Technology, Haifa, Israel; and ${ }^{5}$ Microbiology \\ Laboratory, Rambam Health Care Campus, Haifa, Israel
}

\begin{abstract}
Objective: Israeli hospitals were confronted with a major national outbreak of carbapenemase-producing Enterobacterales (CPE) starting in 2006, caused predominantly by monoclonal Klebsiella pneumoniae carbapenemase (KPC)-producing Klebsiella pneumoniae. Our hospital, Rambam Health Care Campus (RHCC), was one of the medical centers affected by this outbreak. We aimed to investigate the changing epidemiology of CPE at RHCC since 2006.
\end{abstract}

Methods. This was a retrospective observational cohort study performed in Northern Israel (Haifa) at RHCC, which is a primary tertiary acute care academic hospital. The study included all patients who had acquired CPE at RHCC between January 2005 and December 2020.

Results. The proportion of patients infected with K. pneumoniae dropped from 100\% of all CPE in the first years to $28 \%$ (37/134) in 2020. In 2014, the carbapenemase in 94\% of all CPE patients (89/95) was KPC.

\begin{abstract}
Abbreviations: bla, $\beta$-lactamase; CPE, carbapenemase-producing Enterobacterales; IMI, imipenemase; KPC, Klebsiella pneumoniae carbapenemase; MBL, metallo- $\beta$-lactamase; NDM, New Delhi metallo- $\beta$-lactamase; IMI, imipenemase; RHCC, Rambam Health Care Campus; VIM, Verona integron-encoded metallo- $\beta$-lactamase.

Citation: Hussein K, Geffen Y, Eluk O, Warman S, Aboalheja W, Alon T, Firan I, Paul M. The Changing Epidemiology of Carbapenemase-Producing Enterobacterales. Rambam Maimonides Med J 2022;13 (1):e0004. doi:10.5041/RMMJ.10461

Copyright: (C) 2022 Hussein et al. This is an open-access article. All its content, except where otherwise noted, is distributed under the terms of the Creative Commons Attribution License (http://creativecommons.org/licenses/by/3.0), which permits unrestricted use, distribution, and reproduction in any medium, provided the original work is properly cited.
\end{abstract}

Conflict of interest: No potential conflict of interest relevant to this article was reported.

* To whom correspondence should be addressed. E-mail: k_hussein@rambam.health.gov.il 
This decreased to $56 \%$ in 2020, while New Delhi metallo- $\beta$-lactamase (NDM) and OXA- 48 carbapenemases increased from $4 \%$ and $2 \%$ to $29 \%(39 / 134)$ and $12.7 \%$ (17/134) of CPE, respectively.

Conclusions. The CPE epidemic evolved from KPC-producing K. pneumoniae to involve different Enterobacterales and carbapenemases. Our results are a microcosm of the current global epidemiology attesting to globalization in bacteriology. The results have implications for infection control and antibiotic treatment of CPE infections.

KEY WORDS: Carbapenemase-producing Enterobacteriaceae, Enterobacterales, KPC, NDM, OXA-48

\section{INTRODUCTION}

Carbapenem-resistant Enterobacterales (CRE) are amongst the major challenges that have been facing healthcare institutions throughout the world in the last two decades. ${ }^{1}$ Carbapenem resistance among CRE is mediated most commonly by broad-spectrum $\beta$-lactamases that hydrolyze and inactivate the $\beta$ lactam ring of all known $\beta$-lactams, including the last-resort carbapenems. These broad-spectrum enzymes are labeled carbapenemases, and the CRE producing these are named carbapenemaseproducing Enterobacterales (CPE). The most common currently known carbapenemases include Klebsiella pneumoniae carbapenemase (KPC), New Delhi metallo- $\beta$-lactamase (NDM), imipenemase (IMI), oxacillinases (OXA)-48, and Verona integronencoded metallo- $\beta$-lactamase (VIM). Israeli hospitals confronted a major national outbreak starting in 2006, caused predominantly by monoclonal KPC-producing Klebsiella pneumoniae. ${ }^{2}$ Following a national intervention, the spread of CRE was contained. ${ }^{2}$

Our medical center, Rambam Health Care Campus (RHCC), located in Northern Israel, was among the affected hospitals. The prevalence of CRE at RHCC increased from 2006, reaching a peak of 186.6 new acquisitions per 100,000 hospital-days in 2008.3.4 As described nationally, this outbreak was caused by KPC-producing $K$. pneumoniae; from January 2006 to April 2007, all 88 carbapenemresistant $K$. pneumoniae patient isolates carried KPC. 3

Some of the carbapenemases are chromosomal, but most often, and notably, the KPCs are found on plasmids, hence they are able to move between bacterial species. Indeed, with time, KPC spread to different Enterobacterales, and the introduction of diverse carbapenemases was noted. The aim of this study was to describe the introduction of new carba- penemases at RHCC and the spread of carbapenemases among Enterobacterales.

\section{METHODS}

The study was conducted at RHCC, a 1,00o-bed primary and tertiary university hospital in Northern Israel. All patients who acquired CPE at RHCC between January 2005 and December 2020 were included in the study. At RHCC, CPE was considered as acquired if the positive sample was taken $\geq 72$ hours after admission, within 72 hours after any discharge, or within one month after discharge, if the patient was not hospitalized in another healthcare facility. Patients who acquired CRE before admission to RHCC were excluded.

\section{Microbiology}

Rectal swab screening samples were cultured prior to 2019 on PD420 CHROMagar KPC plates; since 2019, samples were cultured on PD517 MSUPERCARBA plates (both from Hy Laboratories Ltd, Rehovot, Israel). The latter medium was used because of its higher sensitivity to non-KPC CPE. Carbapenemase-producing Enterobacterales was defined as Enterobacterales of any type resistant to all tested carbapenems using the Clinical and Laboratory Standards Institute (CLSI) M10oS guidelines definition of MIC $>1$. We extracted DNA from suspected CRE colonies using the Qiamp DNA mini kit (QIAgen, Hilden, Germany) in accordance with the manufacturer's instructions. Since 2014, $\beta$ lactamase (bla) carbapenemase genes, i.e. bla ${ }_{K P C} /$ $b l a_{N D M} / b l a_{O X A-48} / b_{l a}$ VIM , have been detected routinely using polymerase chain reaction (PCR)-based multiplexed assays specific for these genes. 5

\section{RESULTS}

Between January 2005 and December 2020, a total of 891,926 patients were admitted to RHCC. During 
this period, 1,868 patients acquired CPE in RHCC. From 2005 to 2009, almost all isolates were $K$. pneumoniae. Starting in 2009, the proportion of patients carrying CRE with other strains increased gradually, reaching $72 \%(97 / 134)$ in 2020 , while the proportion of K. pneumoniae decreased to $28 \%$ (37/ 134) in 2020 (Figure 1). The most common Enterobacterales other than K. pneumoniae were: Escherichia coli and Enterobacter spp., followed by Citrobacter spp., K. oxytoca, Raoultella spp., Morganella spp., Proteus spp., and Providencia spp.

The mechanisms of resistance and types of carbapenemases also changed. In 2014, 94\% (89/95) of all CPE acquired at RHCC possessed the KPC gene. Only $6 \%(6 / 95)$ of isolates possessed other resistance genes. This proportion decreased to $56 \%$ (75/ 134) in 2020 (Table 1). The proportion of NDM and OXA-48 increased to $29 \%(39 / 134)$ and $12.7 \%$ (17/ 134), respectively (Table 1 ).

\section{DISCUSSION}

We examined the changing epidemiology of CPE in terms of pathogens and resistance mechanisms in one Israeli institution (RHCC) endemic for CPE. Starting from an outbreak confined to KPCproducing K. pneumoniae, spread of carbapenemases to other Enterobacterales strains was reported in RHCC as early as $2010 .{ }^{6}$ In 2020 , only $28 \%$ of all
CPE were K. pneumoniae, and less than half possessed the KPC gene. Carbapenemases spread to many different Enterobacterales, and new carbapenemases were introduced.

Since the first description of KPC-producing $K$. pneumoniae in North Carolina, USA, in 1996,7 KPCproducing $K$. pneumoniae has spread to many locations worldwide. ${ }^{8}$ The KPC gene was subsequently found in other Enterobacteriaceae. ${ }^{9}$ A study using short and long-read sequencing of the carbapenemasecarrying plasmids among Enterobacterales proved plasmid transfer between different Enterobacterales and KPC-containing transposons between plasmids (horizontal transmission), thus explaining the chain of transmission in an outbreak of KPC-producing Enterobacterales in Columbia. ${ }^{10}$ Furthermore, resistance genes have large diversity as described in another study that evaluated the emerging mechanisms of resistance in CRE between 2013 and 2016 at a health system in Northern California, USA. A total of $38.7 \%$ of CRE isolates were carbapenemase genepositive, comprising 25.0\% OXA-48, 20.8\% KPC, 20.8\% NDM, 20.8\% SME, 8.3\% IMP, and 4.2\% VIM. ${ }^{11}$ Although historically the prevalence of carbapenemases had a typical geographic distribution (KPC in USA, Europe, and China; VIM in Greece; OXA-48 in Turkey; and NDM in the Indian subcontinent), these have now spread all over the world. ${ }^{8}$

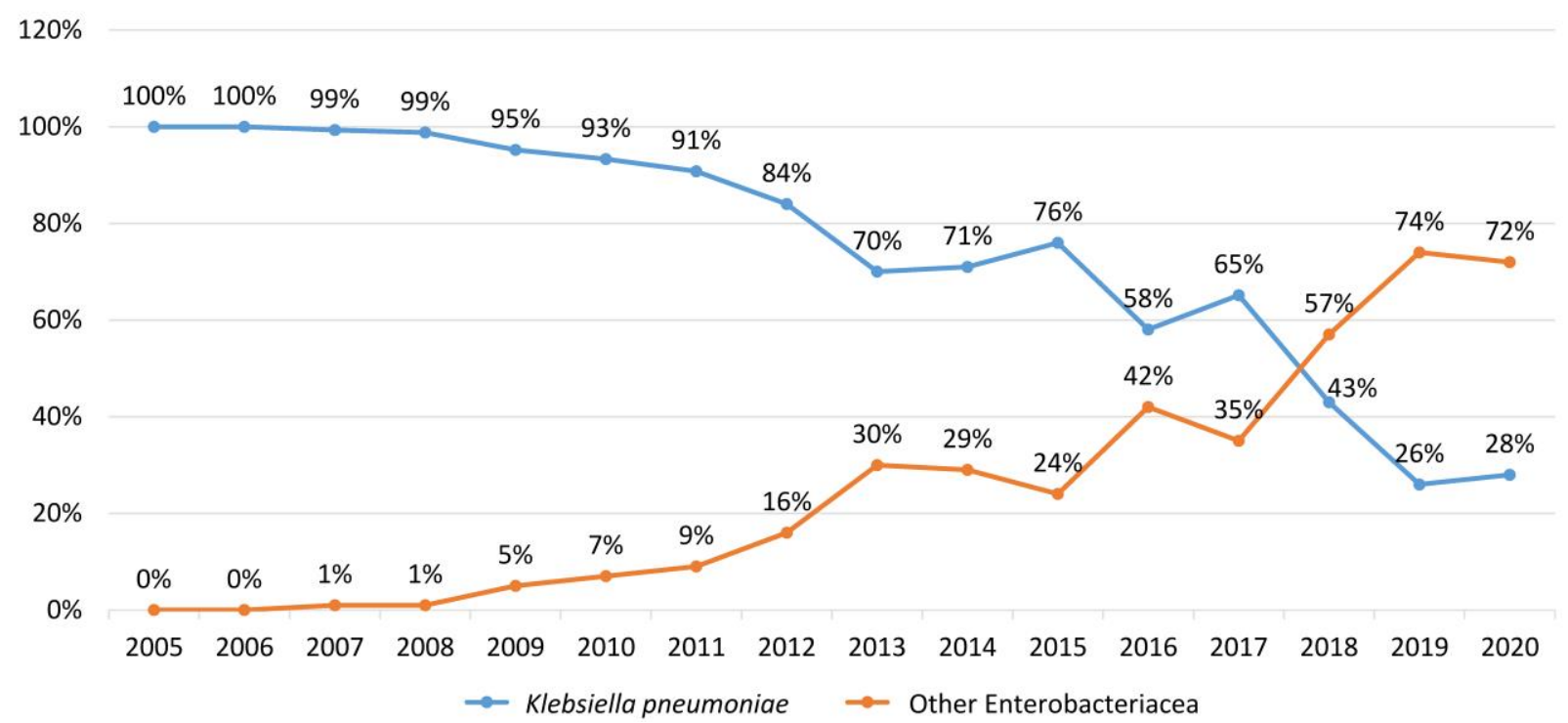

Figure 1. Proportion of CPE Patients diagnosed as carriers of Klebsiella pneumoniae versus with Other Enterobacteriacea at Rambam Health Care Campus, Haifa, Israel 
Table 1. Distribution of CPE Mechanisms of Resistance Among Newly Diagnosed Patients with CPE Per Type and Year at Rambam Health Care Campus, Haifa, Israel.

\begin{tabular}{|c|c|c|c|c|c|}
\hline Year & $\begin{array}{c}\text { Total Number } \\
\text { of CPE } \\
\text { Patients }\end{array}$ & $\begin{array}{c}\text { KPC } \\
n(\%)\end{array}$ & $\begin{array}{c}\text { NDM } \\
n(\%)\end{array}$ & $\begin{array}{c}\text { OXA-48 } \\
n(\%)\end{array}$ & $\begin{array}{c}\text { IMI } \\
n(\%)\end{array}$ \\
\hline 2014 & 95 & $89(94 \%)$ & $4(4 \%)$ & $2(2 \%)$ & 0 \\
2015 & 109 & $100(92 \%)$ & $4(4 \%)$ & $5(5 \%)$ & 0 \\
2016 & 59 & $51(84 \%)$ & $3(5 \%)$ & $5(8 \%)$ & 0 \\
2017 & 65 & $58(88 \%)$ & $4(6 \%)$ & $3(5 \%)$ & 0 \\
2018 & 88 & $58(66 \%)$ & $19(22 \%)$ & $11(13 \%)$ & 0 \\
2019 & 141 & $71(50.5 \%)$ & $41(29 \%)$ & $27(19 \%)$ & $2(1.5 \%)$ \\
2020 & 134 & $75(56 \%)$ & $39(29 \%)$ & $17(12.7 \%)$ & $3(2.3 \%)$ \\
\hline
\end{tabular}

CPE, carbapenemase-producing Enterobacterales; IMI, imipenemase; KPC, Klebsiella pneumoniae carbapenemase; NDM, New Delhi metallo-B-lactamase; OXA, oxacillinases.

Our findings are consistent with this spread, which has a major impact on infection control. The diversity of isolates and resistant genes may indicate that, besides clonal transmission of CRE in the hospital, alternative transmission occurs through horizontal spread of mobile genetic elements and plasmids. Modalities to prevent transfer of carbapenemases between Enterobacterales are necessary, possibly avoiding certain antibiotics, such as carbapenems which are direct drivers of carbapenem resistance. While previously all CRE carriers could be cohorted, separate isolation according to the type of carbapenemase is currently necessary. The diverse resistance mechanisms also affect our choice of empirical and definite antibiotic treatment, since the new $\beta$-lactamases are carbapenemase-specific.

Our study has some limitations. First, it was conducted in one medical center that was endemic for CPE. Second, we included only CPE acquired in RHCC due to lack of microbiological data and molecular studies on isolates acquired elsewhere. However, 95\% of CPE carriers hospitalized in RHCC during the study period had acquired CPE there. This study does not analyze the mechanisms leading to this spread of carbapenemases among bacteria and the reasons leading to the introduction of new carbapenemases to RHCC; only the CPE epidemiology at RHCC over time is described. Similar changes are most likely occurring in other Israeli hospitals. Data published in the 2019 national report for all CPE found in Israeli hospitals reported that only $47 \%$ were KPC, $33 \%$ were NDM, $12 \%$ OXA, $5 \%$ VIM, and $3 \%$ other; it is likely that these CPE circulate within and among hospitals. ${ }^{12}$

In conclusion, we demonstrate the change in carbapenem resistance mechanisms in one Israeli hospital, the introduction of new carbapenemases, and the spread of carbapenemases across Enterobacterales. These findings, if representative of global epidemiology, are of great concern. Irrespective of location, infection control efforts are essential to prevent the further spread of carbapenemases and CPE.

\section{REFERENCES}

1. Nordmann P, Poirel L. The difficult-to-control spread of carbapenemase producers among Enterobacteriaceae worldwide. Clin Microbiol Infect 2014;20:82130. $\underline{\text { CrossRef }}$

2. Schwaber MJ, Lev B, Israeli A, et al. Containment of a country-wide outbreak of carbapenem-resistant Klebsiella pneumoniae in Israeli hospitals via a nationally implemented intervention. Clin Infect Dis 2011;52: 848-55. CrossRef

3. Hussein K, Sprecher H, Mashiach T, Oren I, Kassis I, Finkelstein R. Carbapenem resistance among Klebsiella pneumoniae isolates risk factors, molecular characteristics, and susceptibility patterns. Infect Control Hosp Epidemiol 2009;30:666-71. CrossRef

4. Hussein K, Rabino G, Eluk O, et al. The association between infection control interventions and carbapenemresistant Enterobacteriaceae incidence in an endemic hospital. J Hosp Infect 2017;97:218-25. CrossRef 
5. Monteiro J, Widen RH, Pignatari ACC, Kubasek C, Silbert S. Rapid detection of carbapenemase genes by multiplex real-time PCR. J Antimicrob Chemother 2012;67:906-9. $\underline{\text { CrossRef }}$

6. Geffen Y, Finkelstein R, Oren I, Shalaginov R, Tavleva I, Sprecher H. Changing epidemiology of carbapenemresistant Enterobacteriaceae carriage during an outbreak of carbapenem-resistant Klebsiella pneumoniae. J Hosp Infect 2010;76:355-6. CrossRef

7. Yigit H, Queenan AM, Anderson GJ, et al. Novel carbapenem-hydrolyzing $\beta$-lactamase, KPC-1, from a carbapenem-resistant strain of Klebsiella pneumoniae. Antimicrob Agents Chemother 2001;45:1151-61. CrossRef

8. van Duin D, Doi Y. The global epidemiology of carbapenemase-producing Enterobacteriaceae. Virulence 2017;8:460-9. CrossRef

9. Martirosov DM, Lodise TP. Emerging trends in epidemiology and management of infections caused by carbapenem-resistant Enterobacteriaceae. Diagn Microbiol Infect Dis 2016;85:266-75. CrossRef

10. Rada AM, De La Cadena E, Agudelo C, et al. Dynamics of blaKPC-2 dissemination from non-CG258 Klebsiella pneumoniae to other Enterobacterales via IncN plasmids in an area of high endemicity. Antimicrob Agents Chemother 2020;64:e01743-20. CrossRef

11. Senchyna F, Gaur RL, Sandlund J, et al. Diversity of resistance mechanisms in carbapenem-resistant Enterobacteriaceae at a health care system in Northern California, from 2013 to 2016. Diagn Microbiol Infect Dis 2019;93:250-7. $\underline{\text { CrossRef }}$

12. The Israeli National Center for Infection Control. 2019 Annual Report on Bacterial Alert in General Hospitals. May 24, 2020. Hebrew. Available at: https://www.health.gov.il/UnitsOffice/HD/Infection Control/Documents/IC_Bacterial_alert_2019.pdf (accessed January 4, 2022). 